\title{
RPAS conflict-risk assessment in non-segregated airspace
}

\author{
J.A. Pérez-Castán ${ }^{a, *}$, F. Gómez Comendador ${ }^{a}$, A. Rodríguez-Sanz ${ }^{a}$, I. Armas Cabrera ${ }^{a}$, \\ J. Torrecilla ${ }^{\mathrm{b}}$
}

a Universidad Politécnica de Madrid, Plaza Cardenal Cisneros, 28040 Madrid, Spain

${ }^{\mathrm{b}}$ ISDEFE, Calle Beatriz de Boadilla, 28040 Madrid, Spain

\section{A R T I C L E I N F O}

\section{Keywords:}

Air transport

RPAS

Conflict-risk assessment

Non-segregated airspace

Airspace planning

\begin{abstract}
A B S T R A C T
The efficient and safe introduction of Remotely Piloted Aircraft System (RPAS) in non-segregated airspace requires a thorough assessment of all operational elements. In this paper, we dealt with the safety problem that arises from the integration of RPAS jointly with conventional aircraft in non-segregated airspace. The authors propose a framework and methodology for airspace design and planning purposes based on a conflict-risk method. This method compares a target level of safety (base-scenario without RPAS) with the calculated level of safety (scenario with RPAS). Moreover, the framework proposes an in-depth assessment by identifying geometrical and operational factors that may affect conflict risk. These conflict-risk factors are critical for RPAS integration and must be assessed to detect relations between them and conflict risk. Moreover, we have performed a sensitivity analysis to assess how RPAS average speed affects conflict risk. A real air traffic volume is studied to characterise a conflict-risk indicator, and different permutations to the base-scenario study the RPAS integration all over airways. Results confirm the validity of the methodology for planning purposes and the viability of RPAS integration without a significant impact on safety but with several restrictions to the RPAS airway distribution.
\end{abstract}

\section{Introducción}

The integration of Remotely Piloted Aircraft System (RPAS) into non-segregated airspace is hugely relevant to the Aviation community. In the last years, many regulators, organisations, administrations and researchers have increased their efforts to promote this integration (Dalamagkidis et al., 2008a, 2008b; Oswald and Hershey, 2007; Román-Cordón et al., 2017). Despite the potential benefits of operating RPAS in the European airspace, there is a lack of one standard and consolidated regulation (EASA, 2017, 2015; ICAO, 2015, 2009). Moreover, almost every country has developed their regulation and different barriers to allow operating RPAS in their airspaces. A complex and full of pitfalls regulation do not encourage a free operation of RPAS either the development of the required techniques (Clothier et al., 2011).

Safety is one of the primary concerns preventing progress on RPAS integration. A review of the regulations related to RPAS reveals that administrations have developed a regulatory framework for RPAS operation which demands a risk assessment (EASA, 2017; FAA, 2017). RPAS risk assessment has focused on the evaluation of the risk for thirdparty pedestrians and second-party airspace users (Clothier et al., 2015a, 2015b). In Melnyk et al. (2014) a third-party casualty risk model for RPAS operations is presented to assess the risk to bystander due to RPAS operations. Similar to this work, Clothier et al. (2007) studied a collision-risk model for RPAS to assess the risks to people and property on the ground. However, this ad-hoc safety point of view is visibly individualist and do not contemplate the impact of RPAS integration in non-segregated airspace from the Air Traffic Management (ATM) perspective.

RPAS integration in shared airspace requires a holistic standpoint of the impact on ATM. Regarding the issue of safety, two underlying questions should be tackled beforehand. First, which is an acceptable limit for a safe RPAS operation; and second, how the risk level should be measured.

The first point questions whether the operational concept must be kept or not, i.e., it is required to maintain the same Target level of Safety (TLS) for conventional aviation or to modify it with RPAS inclusions. EASA and FAA assert RPAS introduction must not adversely affect safety (EASA, 2017; FAA, 2017), which means that a TLS must be maintained or reduced but never increased. Several studies estimated the actual safety levels of an RPAS mission (Clothier et al., 2015a, 2015b; Dalamagkidis et al., 2008a, 2008b; Lum and Waggoner, 2011).

\footnotetext{
* Corresponding author.

E-mail address: Javier.perez.castan@upm.es (J.A. Pérez-Castán).
} 


$\begin{array}{ll}\text { Nomenclature } \\ \alpha^{i, j} & \text { angle shaped by airway } i \text { and } j \\ d^{i, j} & \text { critical section at airway } i \text { influenced by airway } j \\ \text { FL } & \text { flight level } \\ H_{\text {min }} & \text { vertical separation minimum } \\ i & \text { airway } \\ j & \text { airway } \\ k & \text { airway continuity } \\ L^{i} & \text { length of the airway } i \\ L_{\text {min }} & \text { longitudinal separation minimum } \\ n & \text { crossing point } \\ N_{C P_{n}}^{i, j} & \text { number of conflicts at crossing point } n \text { for airways } i \text { and } j \\ N_{C P_{n}} & \text { number of conflicts at crossing point } n \text { for every airway } \\ & \text { pars }\end{array}$

NM Nautical Miles

$P_{\exp }^{i} \quad$ exposure probability of an aircraft flying the critical sec-

$P_{C P_{n}}^{i, j} \quad$ conflict probability at crossing point $n$ for airways $i$ and $j$

$P_{C P_{n}} \quad$ conflict probability at crossing point $n$ for every airway pars

$Q^{i} \quad$ aircraft density (number of aircraft per hour) at airway $i$

$Q_{\max }^{i} \quad$ maximum aircraft density (number of aircraft per hour) at airway $i$

$R \quad$ conflict risk per air traffic volume

$t^{i} \quad$ time required to cover the airway $i$

$\tau^{i, j} \quad$ exposure time flying airway $i$ influenced by airway $j$

TLS target level of safety

$\overline{V^{i}} \quad$ average speed of aircraft at airway $i$
These studies attempted to quantify the fatalities caused by RPAS failures, collateral damages overpopulation densities and the TLS required for the mission. The strong point of these methods is that they can ensure a safe level of an individual mission. However, their strong point is as well their weakness because they are not valid methods for an overall analysis of RPAS integration. Realistic and reliable risk methods must be developed or adjusted to allow further integration and development of RPAS in a safe environment.

The second question is more challenging to answer because still there are no agreed-upon risk models to assess airspace planning and design. Since the sixties, different collision risk models have been developed to ensure a safe aircraft operation (Reich, 1966a, 1966b). The International Civil Aviation Organization (ICAO) determines risk models must underlie safety pillars on the subject of a previously characterised TLS (ICAO, 2001, 1998). An in-depth review of risk and safety modelling in civil aviation can be found in (Netjasov and Janic, 2008). In Persiani and Bagassi (2013), the authors proposed a route planner for the integration of RPAS but they focused on the development of conflict detection and resolution algorithms. Most of these methods address to calculate a TLS depending on operational features that affect primary factors of airspace design (Tang et al., 2016). In this way, no previous report on RPAS integration in non-segregated airspace can be found in the literature, and there is no research on explaining how the decision of a fixed TLS affect the number of RPAS that can be introduced in air traffic volume.

The research described in this paper aims to adjust a conflict-risk framework for airspace design for RPAS integration in non-segregated airspace. The goal is to calculate a standard TLS that allows capping the safety level of airspace and assessing how it varies with the introduction of RPAS. It is important to note that the framework in this paper does not develop a new conflict-risk methodology but perform some modifications to the proposed method by Netjasov (2012a). However, what literature is lacking today is the assessment of RPAS integration using a conflict-risk methodology. In this way, a risk and capacity assessment will allow calculating the number of RPAS that can be introduced in an air traffic volume without adverse consequences for safety. A further aim is to assess how the airspace complexity affects the conflict risk depending on operational and geometrical factors as well as to define restrictions for the RPAS introduction. Moreover, a sensitivity analysis focus on assessing how RPAS average speed affects conflict risk. The RPAS introduction is an on-going process which cannot be stopped, and it is unquestionable the need to study the RPAS impact on ATM and to provide the help required for achieving this operational change, finding the balance between capacity and safety.

The rest of the paper is organised as follows. Section 2 presents a brief description of the proposed framework for airspace planning. Section 3 explains the conflict-risk assessment method to assess the impact of RPAS in non-segregated airspace. Section 4 describes the airspace selected, and Section 5 discusses the results and potential restrictions imposed by RPAS operation. Finally, Section 6 draws conclusions and further research.

\section{Framework for RPAS conflict-risk assessment}

The basic idea of this research is to assess how RPAS integration in non-segregated airspace affect safety and conflict risk. RPAS present differences with conventional aircraft in terms of weight, size and operational speed. This research focuses on the integration of heavy RPAS. The most typical RPAS is the RQ4A-Global Hawk with an operational weight over $5000 \mathrm{~kg}$. This type of RPAS is similar to Light aircraft regarding weight but with a lower cruise speed (among 250 and 300 knots) (EUROCONTROL, 2015). The primary goal is to develop a decision-support framework for planning airspace. Hitherto, airspace planning and design processes have borne in mind factors as air traffic control workload and capacity, but few studies have appraised risk or safety.

Airspace planning and design is a high-complexity task that increasingly acquires more relevance in a complex system as air transport. Risk and safety concepts cannot be just ignored and must be gradually integrated at every level of air transport system. In this context, ICAO developed the cornerstone for airspace planning and design with the 9689 Manual (International Civil Aviation Organization, 1998). This manual constitutes the pillar to build a safe air transport system from the airspace design and planning to tactical stage. Particularly, airspace design and planning is (perhaps) the most complex task because it appraises every element and factor of the air traffic system - such as separation minima, airway geometry, traffic density, intervention capacity, communication and surveillance requirements among others. This manual presents a methodology to determine the TLS of an airspace. This TLS assesses whether or not an airspace design fulfils a safety indicator of the air traffic system. The integration of RPAS in non-segregated airspace must ensure that the TLS is not exceeded to ensure safe operations. Therefore, RPAS integration joined-up with conventional aircraft can mean that current airspace planning prevents from this new airspace user.

ICAO's methodology proposes that any concept modification (airspace modification or the introduction of new elements) must be appraised with one of these two approaches: the characterisation of a global TLS which ensure air traffic safety (for instance $10^{-9}$ accidents per flight hour); or the comparison of a safety indicator from a TLS which is considered a reference. This paper follows the second line of action that ascertains a TLS from a real airspace, and the introduction of a new element (RPAS) is compared against it. Hereon, we will determine what are the geometric and operational factors that may favour or inhibit the RPAS introduction and under what conditions.

Finally, this work assumes ideal operational conditions based on a 
planning stage:

- There is an ideal air traffic flow per airway. En-route average speed and aircraft density model air traffic flows. Both operational characteristics are extracted from a trustworthy and accurate database like DDR2 of EUROCONTROL. Besides, traffic information must encompass at least the busiest month of the year, although larger periods improve traffic information.

- The model is focused on an air traffic volume and, mainly, on an enroute scenario where cruise flights encompass the majority of the operations.

- We preclude the impact of climbing/descending flights for further work.

- No deviations from flight planned altitudes and routes or uncertainty variables as wind, temperature or speed are considered.

- Human operator issues are not considered (pilots and air traffic controllers).

\section{Methods}

This section describes the methods used, adjusted or developed to assess the RPAS impact in non-segregated airspace. Firstly, we introduce the conflict-risk assessment method; and secondly, we explain the identification and description of the geometrical and operational factors, which may affect conflict risk.

\subsection{Conflict-risk assessment method}

This paper does not develop an entirely new methodology, but we adjust the conflict-risk assessment method proposed by Netjasov (2012a) with some modifications. Similar to Netjasov's model, Siddiqee (1973) and Geisinger (1985) presented mathematical equations for different conflict situations, delving into the critical section that produces different conflict geometries. Thus, this conflict-risk assessment method aims to determine a TLS depending on operational and geometrical factors. The method is described for a singular Flight Level (FL), but the same equations must be applied for every FL in an air traffic volume. Moreover, the model is focused on en-route scenario where cruise flights encompass the majority of the operations.

In this work, a conflict is defined as the potential situation where two aircraft can infringe the vertical $\left(H_{\min }\right)$ and longitudinal $\left(L_{\min }\right)$ separation minima. The conjunction of the separation minima constitutes a safe volume for each aircraft that cannot be violated at any moment (Alam et al., 2009; Netjasov, 2012b). Every airway intersection (or crossing point) constitutes a critical section, see Fig. 1. The critical section $\left(d^{i, j}\right)$ is located around a crossing point where the path of an aircraft (airway $i$ ) inhibit the entrance of other aircraft (airway $j$ ). Otherwise it may occur a separation minima infringement. A crossing point is the intersection of two airways at least.

The variables that define the critical section are the crossing angle $\left(\alpha^{i, j}\right)$ of two airways involved $(i, j)$ and the horizontal separation minimum $\left(L_{\min }\right)$.

$d^{i, j}=\frac{2 L_{\min }}{\sin \alpha^{i, j}}$

This method is developed for airspace planning purposes. Hence, the operational factors of each airway are the average horizontal speed $\left(\overline{V^{i}}\right)$ and the airway density $\left(Q^{i}\right)$. In this way, we assume that air traffic can be modelled based on constant air traffic flows per each airway. Exposure time $\left(\tau^{i, j}\right)$ is the time required to cover the critical section by an aircraft that operates by the airway $i$, crossing with airway $j$. Moreover, this model requires a first dissection taking into account airway continuity when they concur in a crossing point. This distinction responds to the necessity that arises when an airway ends at a crossing point. The exposure time for these airways without continuity $\left(\tau_{k}^{i, j}\right)$ uses the following expression, where $k$ denotes airways without continuity: $\tau_{k}^{i, j}=\frac{L_{\min }}{\bar{V}^{i} \sin \alpha^{i, j}}$

On the other hand, airways with continuity $\left(\tau^{i, j}\right)$ in a crossing point use the following expression:

$\tau^{i, j}=\frac{2 L_{\min }}{\bar{V}^{i} \sin \alpha^{i, j}}$

The conflict probability of two aircraft flying two airways that concur in a crossing point is the product of the probability of both aircraft are inside their critical section. Considering there are $n$ crossing points in an airspace volume, the conflict probability $\left(P_{C P_{n}}^{i, j}\right)$ for each aircraft pair is:

$P_{C P_{n}}^{i, j}=P_{\text {exp }}^{i} \cdot P_{\text {exp }}^{j}$

where $P_{\exp }^{i}$ and $P_{\exp }^{j}$ is the exposure probability which represents the probability of an aircraft flying at the critical section of airway $i$ and $j$ respectively. This probability is the relation between the time that an aircraft operate inside the critical section and the time $\left(t^{i}\right)$ expended in total length $\left(L^{i}\right)$ of the airway:

$P_{\text {exp }}^{i}=\frac{\tau^{i, j}}{t^{i}} ; P_{\text {exp }}^{j}=\frac{\tau^{j, i}}{t^{j}} ; t^{i}=\frac{L^{i}}{\overline{V^{i}}}$

It is important to note that the conflict probability is calculated for each airway pair that intersects in a crossing point. Thus, if there is a crossing point with more than two airways, the conflict probability $\left(P_{C P_{n}}\right)$ at the crossing point $n$ is calculated by the addition of every airway pair:

$P_{C P_{n}}=\sum_{i \neq j} P_{C P_{n}}^{i, j}$

Therefore, conflict probability at each crossing point depends on its geometry, the number of airways that coalesce and the aircraft average horizontal speed for each airway.

The last step to calculate the number of conflict at a crossing point requires considering the air traffic flow at each airway. The airway density $\left(Q^{i}\right)$ represents the number of aircraft per hour that operates through the airway $i$. This airway density is calculated by the average density of the busiest periods per day. This variable is crucial to assess the number of conflict occurrences that can occur because it introduces the concept of severity. A high-traffic density may imply a high number of conflicts depending on the air traffic flow of the rest of airways. This idea introduces a new concept: effective crossing points. Effective crossing points are those crossing points that present air traffic flow for at least two crossing airways, i.e., an intersection where solely one airway adds traffic cannot be considered a crossing point because there are no aircraft to collide with. This condition is ascertained by the airways with an air traffic flow different from zero $(Q \neq 0)$.

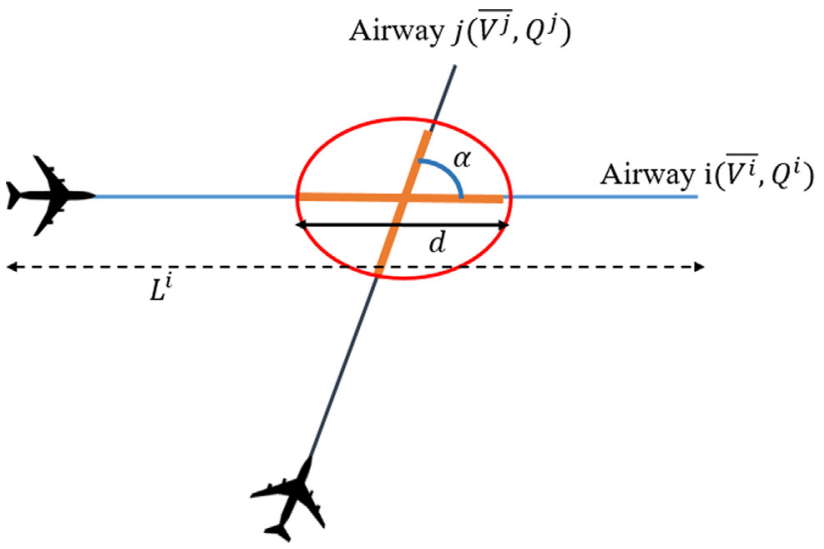

Fig. 1. Representation of geometrical and operational variables in a crossing point. The critical section is the ellipse around a crossing point. 
Typically, risk is defined as the product or combination of probability and severity. Herein, probability refers to the conflict probability, and severity refers to the number of aircraft that can collide at a crossing point, i.e., severity is the product of airway density involved $\left(Q^{i} Q^{j}\right)$. Hence, conflict risk refers to the number of conflicts in an air traffic volume. The total number of conflicts $\left(N_{C P_{n}}\right)$ at each crossing point is the addition of all the possible combinations of airway pairs $\left(N_{C P_{n}}^{i, j}\right)$ :

$N_{C P_{n}}^{i, j}=Q^{i} Q^{j} P_{C P_{n}}^{i, j}$

$N_{C P_{n}}=\Sigma_{i \neq j} N_{C P}^{i, j}$

Finally, the total conflict risk $(R)$ of an air traffic volume is the addition of the individual conflict risk at every crossing point (Mehadhebi and Lazaud, 2004):

$R=\sum_{1}^{n} N_{C P n}$

The value of the $R$ under normal conditions ascertains the limit of TLS. TLS is the boundary with that we can assess the impact of RPAS introduction in a non-segregated airspace.

\subsection{Conflict-risk factors}

One aim of this study is to calculate the maximum number of RPAS that can be introduced in an air traffic volume without the increment of conflict risk. To achieve this goal, it is need to detect what factors influence on the conflict risk. Simulations with RPAS are performed by substituting the conventional air traffic flow by RPAS air traffic flow. This RPAS air traffic flow introduces modifications to average horizontal speed (RPAS operate at slower speeds) and airway density (simulations looks at the maximum number of RPAS without exceeding the TLS).

Attending the methodology proposed, there are three types of conflict-risk factors: morphological, geometrical and operational.

Table 1 identifies the primary factors that affect conflict risk.

Morphological and geometrical factors are fixed because they are specified by airspace design, although it varies depending on the air traffic volume. Conversely, operational factors can suffer variations. These variations in air traffic flows depend on the operational day or the period of study considered. Apart from that, RPAS speed depends on the aircraft type and operational performances. This study focuses on RPAS that can operate in the upper airspace. However, RPAS operational performances are lower than conventional aircraft. Previous studies (Clothier et al., 2015a, 2015b; EUROCONTROL, 2015; PérezCastán et al., 2017) showed that RQ4A-Global Hawk and MQ9-Reaper are the most suitable RPAS to operate jointly with conventional aircraft.
Table 2

RPAS average horizontal speed for sensitivity analysis.

\begin{tabular}{llll}
\hline & Experiment 1 & Experiment 2 & Experiment 3 \\
\hline $\begin{array}{c}\text { RPAS Average horizontal speed } \\
\text { (knots) }\end{array}$ & 250 & 300 & 350 \\
\hline
\end{tabular}

Furthermore, we have performed a sensitivity analysis to assess how RPAS average speed affects conflict risk, without modifying others conflict-risk factors. Table 2 characterises the experiments considered.

\section{Scenario description}

This section describes the air traffic volume selected to apply the methodology of the previous section. The air traffic volume selected is LECMPAU located at Madrid upper airspace (Spain). The reason for this sector is because it presents a high number of cruise flights between FL 250-310. These FLs are chosen to assess the RPAS integration, and we have collected real traffic data of this sector in August 2017. Overall, LECMPAU presents 25 airways and 55 crossing points although not every airway has air traffic nor every crossing points will contribute to the conflict risk. Fig. 2 represents LECMPAU from two points of view: the left picture highlights the airways that have traffic, and the right picture highlights the crossing points.

The study of conflict-risk factors considered in Section 3.2 allows assessing the scenario in detail:

- Number of crossing points: Airway distribution in LECMPAU volume generates 55 crossing points. However, 14 airways do not have traffic that means the disappearance of 42 crossing points, i.e., solely 13 crossing points are effective to assess (see Fig. 3).

- Crossing angle: the crossing angle distribution is very diverse in this air traffic volume where there are airways that intersect almost perpendicular and others minor than $30^{\circ}$. In this way, we separate the crossing angle in three groups from assessing the influence of this factor: $\alpha<30^{\circ}, 30^{\circ}<\alpha<60^{\circ}, 60^{\circ}<\alpha<90^{\circ}$.

- Airway length: Airway length in LECMPAU varies from UQ300 (632 Nautical Miles - NM) to UN10 or UT430 (20 NM). Except for the shortest and largest airways, most of the airways present the same order of magnitude. We classify them into three groups: Large ( $L>400 \mathrm{NM})$, Medium $(200<L<400 \mathrm{NM})$ and Short $(L<200 \mathrm{NM})$.

- Airway density: LECMPAU have air traffic in 11 of 25 airways, and their traffic density is diverse: there are airways with a density from 0.045 to 4 aircraft per hour.

- Conventional aircraft speed: Average horizontal speed of

Table 1

Description of conflict-risk factors.

Morphological factors

Number of crossing points

Geometrical factors

Crossing angle

Airway Length

Operational factors

Airway density

RPAS speed
Although the relation between some crossing points in an air traffic volume and the total conflict risk is not linear, there is an evident influence on the number of crossing points and the risk. Hence, the higher number of crossing points, the higher conflict risk in an air traffic volume. In turn, those airways with a higher number of crossing points will presumably present a major conflict risk

Every crossing point has its geometrical features. Particularly, the crossing angle of each crossing point is a crucial factor in determining the exposure time. There are airway geometries that promote safety (angles close to $90^{\circ}$ ) and others that do not (angles minor than $30^{\circ}$ ) If an aircraft expends more time in an airway, it will imply minor conflict risk. This assentation, which seems illogical, has its reasoning in the exposure probability definition $\left(P_{\text {exp }}^{i}\right)$ of Eq. (5). The exposure probability is the ratio between the exposure time $\left(\tau^{i, j}\right)$ and the flight time in the airway $\left(t_{i}\right)$. Thus, if $\downarrow t_{i}$ with $\tau^{i, j}=$ constant $\rightarrow P_{\text {exp }}^{i} \uparrow$

Airway density is a crucial factor because higher air traffic per airway means a higher number of conflicts as well as conflict risk. Nonetheless, results will be conditioned by the number of conventional aircraft removed

The TLS calculated depends on the average horizontal speed of the aircraft that operate in the airways. To remove conventional traffic and introduce RPAS in an airway imply a drastic modification on the average horizontal speed that may affect the conflict risk. On the whole, the slower the horizontal aircraft speed, the higher is the exposing time in a critical section and, in turn, the higher is the conflict probability 

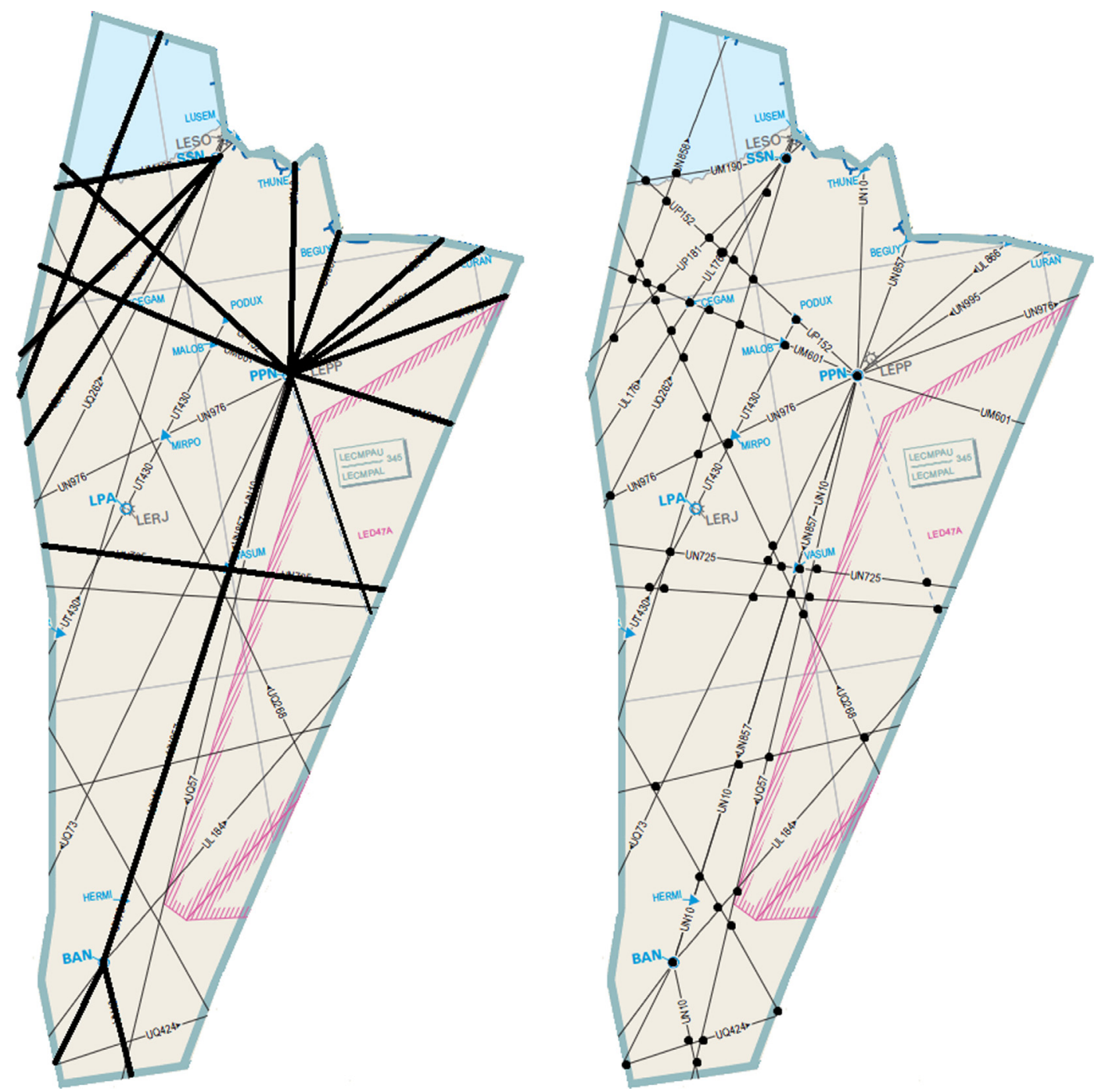

Fig. 2. LECMPAU sector: At the (left) Airways with traffic during the period of study, and at the (right) Crossing points detected.

conventional aircraft is assumed to be constant, and in the majority of the airways aircraft fly around 400 knots.

Lastly, the longitudinal separation minimum in the Spanish upper airspace is $5 \mathrm{NM}$.

\section{Results and discussions}

The methodology proposed is applied in the scenario described in the previous section. Firstly, we describe the results for the base-scenario to characterise the TLS that cap the conflict risk with RPAS. Secondly, we present the results for the base-scenario modified by the introduction of RPAS. Results based on RPAS integration are separated in two sections: the first analysis assesses how the conflict risk varies regarding the number of RPAS introduced per airway; and later on, we assess the variation of the conflict-risk factors and the consequences to formulate regulations for RPAS integration in non-segregated airspace.

\subsection{Base-scenario: TLS definition}

The base-scenario was described in Section 3. The conflict-risk assessment aims to characterise a TLS which could be compared with RPAS. Table 3 shows the conflict risk results calculated for each crossing point between FL 250-310:

Solely $24 \%$ (13 of 55 ) of the crossing points contribute to the conflict risk because of the high number of airways without air traffic. These 13 crossing points are depicted in Fig. 3 with a thickness scale to represent the influence of each crossing point to the total conflict risk. There is a considerable variation between conflict-risk values of the 13 crossing points: 7 presents a conflict risk extremely low (E-06); 4 with a conflict risk low (E-04); and 2 with a high conflict risk (E-02). $C P_{11}$ and $C P_{50}$ are the riskier crosing points. They present the worst combination of conflict-risk factors - such as the higher number of airways concurring at them and the airways with the higher air traffic density. Therefore, the critical elements for the sector LECMPAU are the crossing points $C P_{11}$ and $C P_{50}$. Lastly, the sum of the whole crossing points contribution to the total conflict risk volume produces the TLS:

$T L S=R=0,020512$

The total conflict risk value (TLS) is specific for this sector, the FLs considered and the period of study. This TLS is not a fixed value for every sector, but it is a representative value of a scenario under specific operational conditions. Therefore, it must be considered only for relative assessments.

\subsection{RPAS introduction}

This section shows the conflict-risk results by the introduction of RPAS in LECMPAU. The process of RPAS simulation is to remove conventional aircraft for each airway and to introduce RPAS with their 


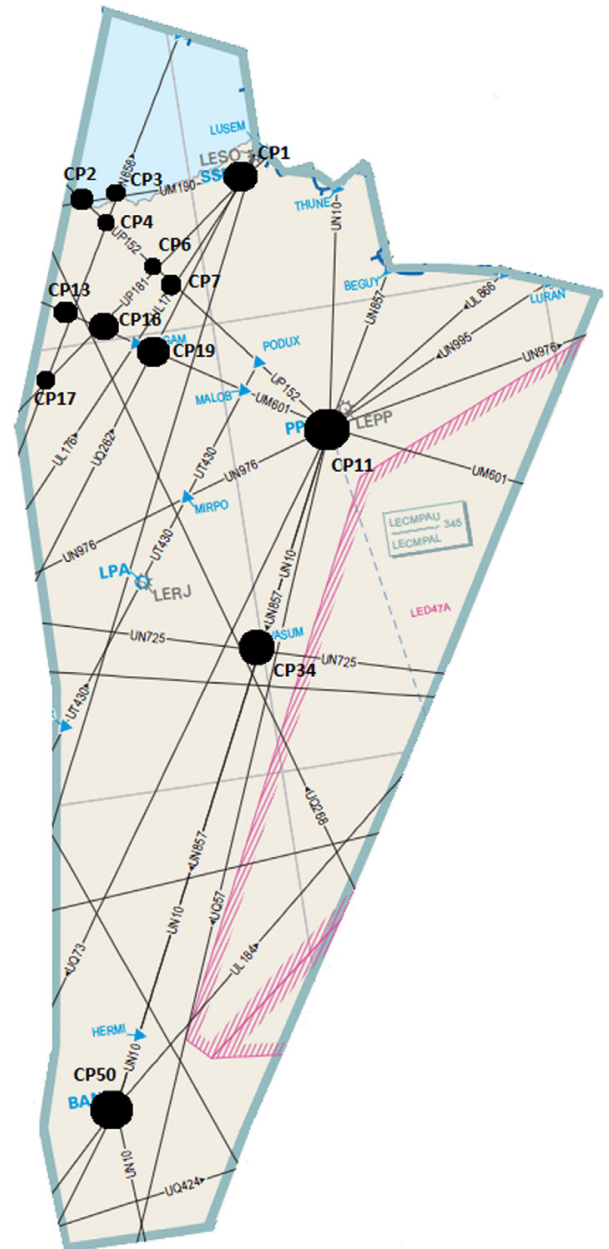

Fig. 3. Crossing points that constitute the conflict risk. The thickness of the circles represents the relative influence on the conflict risk.

Table 3

Conflict risk per crossing point.

\begin{tabular}{ll}
\hline Crossing points & $\boldsymbol{R}_{\boldsymbol{C P} \boldsymbol{n}}$ \\
\hline$C P_{1}$ & $3,94 \mathrm{E}-05$ \\
$C P_{2}$ & $3,88 \mathrm{E}-06$ \\
$C P_{3}$ & $2,04 \mathrm{E}-06$ \\
$C P_{4}$ & $4,96 \mathrm{E}-07$ \\
$C P_{6}$ & $4,95 \mathrm{E}-07$ \\
$C P_{7}$ & $2,27 \mathrm{E}-06$ \\
$C P_{11}$ & $1,98 \mathrm{E}-02$ \\
$C P_{13}$ & $6,13 \mathrm{E}-06$ \\
$C P_{16}$ & $1,28 \mathrm{E}-05$ \\
$C P_{17}$ & $1,07 \mathrm{E}-06$ \\
$C P_{19}$ & $2,50 \mathrm{E}-05$ \\
$C P_{34}$ & $1,04 \mathrm{E}-4$ \\
$C P_{50}$ & $1,05 \mathrm{E}-2$ \\
$R$ & $2,05 \mathrm{E}-2$ \\
\hline
\end{tabular}

operational characteristics, i.e., we exchange the conventional airway density and ground speed with new RPAS airway density and RPAS ground speed. Each simulation increases the number of RPAS considered until the CLS exceeds the TLS. Furthermore, a sensitivity analysis assesses the impact of RPAS speed variations on the conflict risk.

\subsubsection{RPAS sensitivity analysis}

Once we have determined the conflict risk TLS for this study, the methodology is applied but introducing RPAS through the airways. Tables 4-6 present conflict-risk values calculated for each experiment.
Each column denotes the airway that its conventional traffic has been removed and each row indicates the number of RPAS introduced. Besides, the first row introduces the initial traffic, i.e., the airway density with conventional aircraft, and each cell provides the conflict risk of the air traffic volume with this operational features. Grey background cells highlight the combinations (number of RPAS per airway) that provide a conflict risk minor than the TLS, and, therefore, they could afford the introduction of this RPAS density. Finally, the maximum number of RPAS allowed is summarised at the end of each table.

Table 4 shows the results of experiment 1 , with the minor average horizontal speed for RPAS (250 knots). The maximum number of RPAS than can be introduced for this horizontal speed is 10 in the airway UM190. This airway jointly with UN725 and UP152 provide the minor conflict risk. Regarding airway density, UM190 and UP152 are routes with low initial traffic, but UN725 presents high traffic. Therefore, we cannot confirm that the initial traffic is a limiting factor for the election of critical airways. Apart from that, 15 airways do not allow the introduction of any RPAS (12 of them do not have initial traffic). This condition means a restriction because the RPAS integration through any of these 12 airways (UQ262, UM176, UQ148, UQ57, UQ73, UT430, UQ400, UQ88, UL184, UQ424, UQ300 and UQ268) implies an increment on the number of conflicts and the conflict risk. There are other three airways (UN858, UN995 and UP181) that do not allow RPAS. These particular airways have initial traffic and must be assessed indepth to understand the reason why they do not accept RPAS.

Table 5 shows the results of experiment 2 where the average horizontal speed of RPAS is 300 knots. The increase of the average horizontal speed means an increment of the maximum number of RPAS allowed in the air traffic volume. The speed increment permits to introduce up to 12 RPAS in UM190, which means an increase of RPAS by 2. This behaviour is not limited to this airway, but it is suitable for the rest of the airways with initial traffic, for instance, UN725 increases from 8 to 10 and UP152 from 6 to 7. On the other hand, airways which do not permit any RPAS in experiment 1 still do not allow change. Nonetheless, the three particular airways mentioned previously can introduce one RPAS due to the speed increment.

Finally, Table 6 shows the results of experiment 3 with the fastest RPAS average horizontal speed (350 knots). These results confirm the outcomes of experiment 1 and 2. Airways with initial traffic allow RPAS introduction, and the number of RPAS (without exceeding TLS) maintains the same behaviour regarding speed increment: higher speed means a major number of RPAS. In this experiment, the maximum number of RPAS increases up to 14 in UM190. On the other hand, airways without initial traffic still do not allow the introduction of RPAS.

Therefore, these results conclude that the suppression of initial traffic for RPAS integration is feasible and can achieve a number of RPAS near to the initial traffic or even to surpass it. Solely airway UP181 and UL176 do not confirm this outcome, and more analysis is required to detect which are the underlying reasons: for instance, in our study, we only have considered entire numbers of RPAS while the initial traffic does not have to. On the other hand, airways without initial traffic do not allow any RPAS introduction. Furthermore, RPAS horizontal speed is a crucial factor because greater average horizontal speed means a higher number of RPAS through the air traffic volume. Lastly, one limitation of this work is that the methodology applied does not allow considering a mix of RPAS and conventional aircraft per airway.

\subsubsection{Assessment of conflict-risk factors}

The assessment aims to delve into the behaviour of conflict-risk factors and determine, as far as possible, requirements for RPAS introduction in non-segregated airspace, i.e., how conflict-risk factors affect and which of them are critical. However, one limitation of this analysis is that it cannot accurately ensure which the primary causes are. The reason is that it can exist combinations between different 
Table 4

Variation of conflict risk by the introduction of RPAS with 250 knots.

UL176 UL866 UM190 UM601 UN10 UN725 UN857 UN858 UN976 UN995 UP152 UP181 UQ268, UQ300, UQ400, UQ424,

UQ57, UQ73, UQ88, UT430

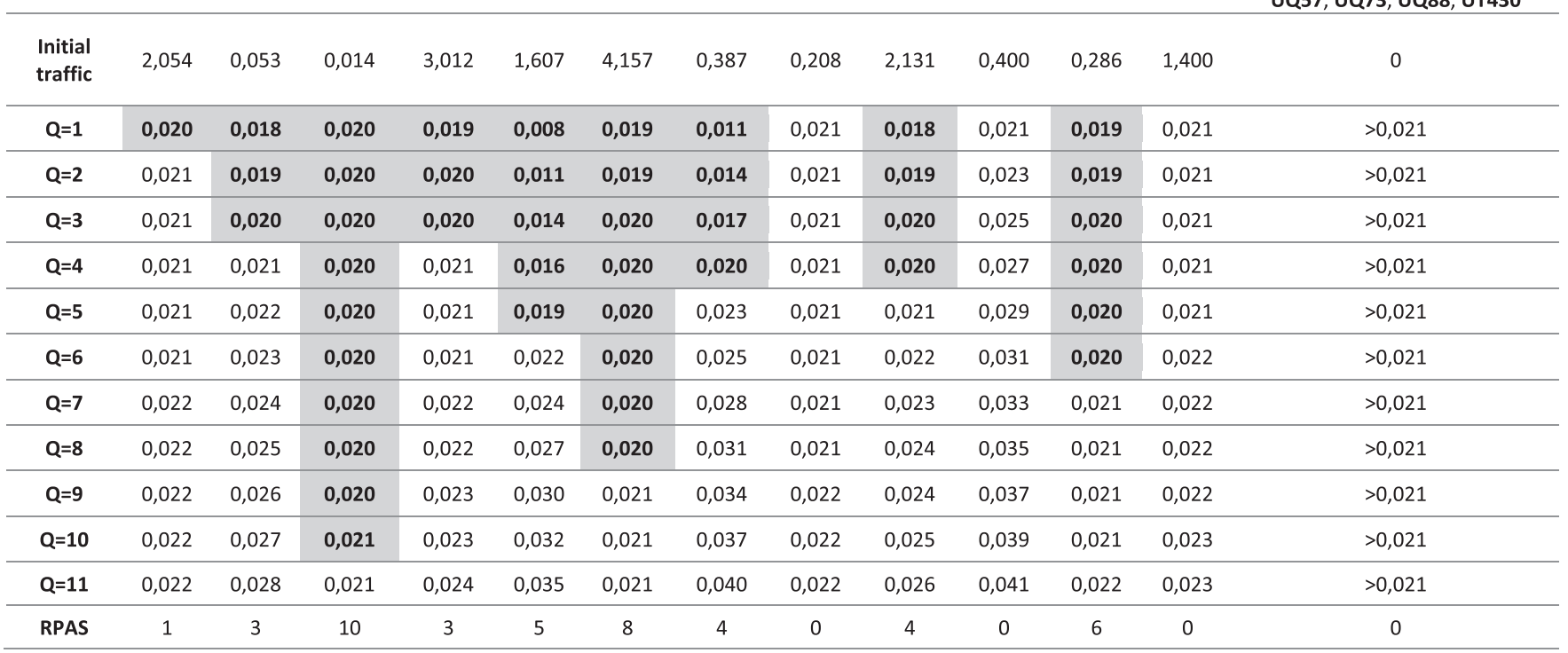

factors or perhaps the existence of other factors that are not considered herein. For the sake of clarity, conflict-risk factors assessment is performed based on one single RPAS with an average horizontal speed of 300 knots.

Table 7 shows the results for different conflict-risk factors. Graphically, Table 7 is sorted depending on the total conflict risk of LECMPAU where one RPAS flies. The second column shows the total conflict risk that supposes the introduction of one RPAS for each airway.

Attending to the total conflict risk, airways can be grouped into three groups. These three groups are based on a relative order where low conflict risk is lower than 0.02 , and high conflict-risk refers to the highest conflict-risk airways.
1. High conflict risk: Airways UQ57 $(0,0226)$ and UL184 $(0,0214)$ presents the highest total conflict risk. These airways do not have initial traffic, and the introduction of one RPAS provides the worst results. Their characteristics are very diverse considering conflictrisk factors, and there is no a definite primary factor but a combination of them.

2. Medium conflict risk: Airways from UT430 (0,0210) to UM190 $(0,0202)$ presents medium values of total conflict risk. These airways present a variation of conflict risk less than $5 \%$ among them. In this group, there are airways with and without initial traffic. However, the influence of the existence or not of initial traffic is clear because airways that fulfil that condition provide higher

Table 5

Variation of conflict risk by the introduction of RPAS with 300 knots. UL176 UL866 UM190 UM601 UN10 UN725 UN857 UN858 UN976 UN995 UP152 UP181 UQ268, UQ300, UQ400, UQ424, UQ57, UQ73, UQ88, UT430

\begin{tabular}{|c|c|c|c|c|c|c|c|c|c|c|c|c|c|}
\hline & & & & & & & & & & & & & Q/3, UQ88, U/430 \\
\hline $\begin{array}{l}\text { Initial } \\
\text { traffic }\end{array}$ & 2,054 & 0,053 & 0,014 & 3,012 & 1,607 & 4,157 & 0,387 & 0,208 & 2,131 & 0,400 & 0,286 & 1,400 & 0 \\
\hline$Q=1$ & 0,020 & 0,018 & 0,020 & 0,019 & 0,008 & 0,019 & 0,010 & 0,020 & 0,018 & 0,020 & 0,019 & 0,020 & $>0,021$ \\
\hline$Q=2$ & 0,021 & 0,019 & 0,020 & 0,020 & 0,010 & 0,019 & 0,013 & 0,021 & 0,018 & 0,022 & 0,019 & 0,021 & $>0,021$ \\
\hline$Q=3$ & 0,021 & 0,020 & 0,020 & 0,020 & 0,012 & 0,020 & 0,015 & 0,021 & 0,019 & 0,024 & 0,020 & 0,021 & $>0,021$ \\
\hline$Q=4$ & 0,021 & 0,021 & 0,020 & 0,020 & 0,015 & 0,020 & 0,018 & 0,021 & 0,020 & 0,025 & 0,020 & 0,021 & $>0,021$ \\
\hline$Q=5$ & 0,021 & 0,022 & 0,020 & 0,021 & 0,017 & 0,020 & 0,020 & 0,021 & 0,020 & 0,027 & 0,020 & 0,021 & $>0,021$ \\
\hline$Q=6$ & 0,021 & 0,022 & 0,020 & 0,021 & 0,019 & 0,020 & 0,023 & 0,021 & 0,021 & 0,029 & 0,020 & 0,021 & $>0,021$ \\
\hline$Q=7$ & 0,021 & 0,023 & 0,020 & 0,021 & 0,021 & 0,020 & 0,025 & 0,021 & 0,022 & 0,031 & 0,020 & 0,022 & $>0,021$ \\
\hline$Q=8$ & 0,022 & 0,024 & 0,020 & 0,022 & 0,024 & 0,020 & 0,027 & 0,021 & 0,022 & 0,032 & 0,021 & 0,022 & $>0,021$ \\
\hline$Q=9$ & 0,022 & 0,025 & 0,020 & 0,022 & 0,026 & 0,020 & 0,030 & 0,021 & 0,023 & 0,034 & 0,021 & 0,022 & $>0,021$ \\
\hline$Q=10$ & 0,022 & 0,026 & 0,020 & 0,022 & 0,028 & 0,021 & 0,032 & 0,021 & 0,024 & 0,036 & 0,021 & 0,022 & $>0,021$ \\
\hline$Q=11$ & 0,022 & 0,027 & 0,020 & 0,023 & 0,030 & 0,021 & 0,035 & 0,022 & 0,024 & 0,038 & 0,021 & 0,022 & $>0,021$ \\
\hline$Q=12$ & 0,022 & 0,027 & 0,021 & 0,023 & 0,032 & 0,021 & 0,037 & 0,022 & 0,025 & 0,039 & 0,021 & 0,023 & $>0,021$ \\
\hline$Q=13$ & 0,022 & 0,028 & 0,021 & 0,023 & 0,035 & 0,021 & 0,040 & 0,022 & 0,026 & 0,041 & 0,022 & 0,023 & $>0,021$ \\
\hline RPAS & 1 & 3 & 12 & 4 & 6 & 10 & 5 & 1 & 5 & 1 & 7 & 1 & 0 \\
\hline
\end{tabular}


Table 6

Variation of conflict risk by the introduction of RPAS with 350 knots.

UL184, UM176, UQ148, UQ262, UL176 UL866 UM190 UM601 UN10 UN725 UN857 UN858 UN976 UN995 UP152 UP181 UQ268, UQ300, UQ400, UQ424,

\begin{tabular}{|c|c|c|c|c|c|c|c|c|c|c|c|c|c|}
\hline & & & & & & & & & & & & & Q73, UQ88, UT430 \\
\hline $\begin{array}{l}\text { Initial } \\
\text { traffic }\end{array}$ & 2,054 & 0,053 & 0,014 & 3,012 & 1,607 & 4,157 & 0,387 & 0,208 & 2,131 & 0,400 & 0,286 & 1,400 & 0 \\
\hline$Q=1$ & 0,020 & 0,018 & 0,020 & 0,019 & 0,008 & 0,019 & 0,010 & 0,020 & 0,018 & 0,020 & 0,019 & 0,020 & $>0,021$ \\
\hline$Q=2$ & 0,021 & 0,019 & 0,020 & 0,020 & 0,010 & 0,019 & 0,012 & 0,021 & 0,018 & 0,022 & 0,019 & 0,021 & $>0,021$ \\
\hline$Q=3$ & 0,021 & 0,019 & 0,020 & 0,020 & 0,011 & 0,020 & 0,014 & 0,021 & 0,019 & 0,023 & 0,020 & 0,021 & $>0,021$ \\
\hline$Q=4$ & 0,021 & 0,020 & 0,020 & 0,020 & 0,013 & 0,020 & 0,016 & 0,021 & 0,019 & 0,025 & 0,020 & 0,021 & $>0,021$ \\
\hline$Q=5$ & 0,021 & 0,021 & 0,020 & 0,020 & 0,015 & 0,020 & 0,018 & 0,021 & 0,020 & 0,026 & 0,020 & 0,021 & $>0,021$ \\
\hline$Q=6$ & 0,021 & 0,022 & 0,020 & 0,021 & 0,017 & 0,020 & 0,020 & 0,021 & 0,021 & 0,027 & 0,020 & 0,021 & $>0,021$ \\
\hline$Q=7$ & 0,021 & 0,022 & 0,020 & 0,021 & 0,019 & 0,020 & 0,023 & 0,021 & 0,021 & 0,029 & 0,020 & 0,021 & $>0,021$ \\
\hline$Q=8$ & 0,021 & 0,023 & 0,020 & 0,021 & 0,021 & 0,020 & 0,025 & 0,021 & 0,022 & 0,030 & 0,020 & 0,022 & $>0,021$ \\
\hline$Q=9$ & 0,022 & 0,024 & 0,020 & 0,022 & 0,023 & 0,020 & 0,027 & 0,021 & 0,022 & 0,032 & 0,021 & 0,022 & $>0,021$ \\
\hline$Q=10$ & 0,022 & 0,025 & 0,020 & 0,022 & 0,025 & 0,020 & 0,029 & 0,021 & 0,023 & 0,033 & 0,021 & 0,022 & $>0,021$ \\
\hline$Q=11$ & 0,022 & 0,025 & 0,020 & 0,022 & 0,027 & 0,020 & 0,031 & 0,021 & 0,023 & 0,035 & 0,021 & 0,022 & $>0,021$ \\
\hline$Q=12$ & 0,022 & 0,026 & 0,020 & 0,023 & 0,029 & 0,021 & 0,033 & 0,021 & 0,024 & 0,036 & 0,021 & 0,022 & $>0,021$ \\
\hline$Q=13$ & 0,022 & 0,027 & 0,020 & 0,023 & 0,031 & 0,021 & 0,035 & 0,022 & 0,025 & 0,038 & 0,021 & 0,022 & $>0,021$ \\
\hline$Q=14$ & 0,022 & 0,027 & 0,021 & 0,023 & 0,032 & 0,021 & 0,037 & 0,022 & 0,025 & 0,039 & 0,021 & 0,023 & $>0,021$ \\
\hline$Q=15$ & 0,022 & 0,028 & 0,021 & 0,023 & 0,034 & 0,021 & 0,039 & 0,022 & 0,026 & 0,041 & 0,022 & 0,023 & $>0,021$ \\
\hline RPAS & 1 & 4 & 14 & 5 & 7 & 11 & 6 & 1 & 5 & 1 & 8 & 1 & 0 \\
\hline
\end{tabular}

conflict risk that airways with initial traffic. Their characteristics follow the trend that the number of crossing points increases as well as the airway length and the number of crossing points with lower crossing angles.

3. Low conflict risk: Airways from UM601 $(0,0193)$ to UN10 $(0,008)$ presents the lowest total conflict risk. Their characteristics are evident because the introduction of RPAS do not suppose the appearance of new crossing points and they share the same geometrical characteristics. The conflict-risk variance is due to the variation of operational factors: between initial traffic and number of RPAS introduced.

5.2.2.1. Number of crossing points. The number of crossing points in an air traffic volume is fixed. However, the number of effective crossing points depends on the operational characteristics, determined by airway density. Thus, RPAS introduction implies two possible

Table 7

Results disaggregated depending on conflict-risk factors.

\begin{tabular}{|c|c|c|c|c|c|c|}
\hline Airway & $\mathrm{R}$ & No of Crossing points & $\begin{array}{l}\text { No of crossing points with } \\
\alpha<30^{\circ}\end{array}$ & $\begin{array}{l}\text { No of crossing points with } \\
30^{\circ}<\alpha<60^{\circ}\end{array}$ & $\begin{array}{l}\text { No of crossing points with } \\
60^{\circ}<\alpha<90^{\circ}\end{array}$ & Airway length \\
\hline UQ57 & 0,0226 & 15 & 21 & 10 & 11 & MEDIUM \\
\hline UL184 & 0,0214 & 13 & 17 & 7 & 11 & SHORT \\
\hline UT430 & 0,0210 & 17 & 20 & 8 & 9 & SHORT \\
\hline UQ73 & 0,0209 & 14 & 20 & 9 & 12 & LARGE \\
\hline UM176 & 0,0207 & 14 & 21 & 9 & 11 & SHORT \\
\hline UQ262 & 0,0206 & 17 & 20 & 7 & 12 & LARGE \\
\hline UQ268 & 0,0206 & 20 & 19 & 12 & 9 & LARGE \\
\hline UQ148 & 0,0205 & 17 & 20 & 8 & 11 & MEDIUM \\
\hline UQ88 & 0,0205 & 14 & 17 & 8 & 9 & LARGE \\
\hline UQ300 & 0,0205 & 14 & 17 & 8 & 9 & LARGE \\
\hline UQ424 & 0,0205 & 15 & 19 & 7 & 9 & LARGE \\
\hline UQ400 & 0,0205 & 14 & 18 & 7 & 9 & LARGE \\
\hline UN858 & 0,0205 & 13 & 17 & 7 & 9 & SHORT \\
\hline UP181 & 0,0205 & 13 & 17 & 7 & 9 & SHORT \\
\hline UL176 & 0,0204 & 13 & 17 & 7 & 9 & SHORT \\
\hline UN995 & 0,0203 & 13 & 17 & 7 & 9 & SHORT \\
\hline UM190 & 0,0202 & 13 & 17 & 7 & 9 & SHORT \\
\hline UM601 & 0,0193 & 13 & 17 & 7 & 9 & SHORT \\
\hline UN725 & 0,0193 & 13 & 17 & 7 & 9 & SHORT \\
\hline UP152 & 0,0192 & 13 & 17 & 7 & 9 & SHORT \\
\hline UL866 & 0,0182 & 13 & 17 & 7 & 9 & SHORT \\
\hline UN976 & 0,0178 & 13 & 17 & 7 & 9 & SHORT \\
\hline UN857 & 0,0104 & 13 & 17 & 7 & 9 & SHORT \\
\hline UN10 & 0,008 & 13 & 17 & 7 & 9 & SHORT \\
\hline
\end{tabular}


situations:

(a) The introduction of RPAS supplants conventional traffic from an airway: the number of crossing points remains unchangeable.

(b) The introduction of RPAS does not remove conventional traffic because there was not conventional traffic previously: the number of crossing points increases.

The third column of Table 7 shows how this conflict-risk factor varies with RPAS introduction. It is evident that scenarios with a minor number of crossing points (case a) provide minor total conflict risk. Thus, the first recommendation is that RPAS introduction should not mean the appearance of new crossing points in the air traffic volume because it increases the conflict risk. The implications are severe because the operational sequences imply that RPAS should fly airways which have initial traffic. Nonetheless, there exist exceptions at UL184 and UT430 airways. Despite RPAS operation at these airways do not provide a high increment on crossing points, the total conflict risk climbs up to the second and the third position. This behaviour cannot be explained based on the number of crossing points, but it requires further assessment.

5.2.2.2. Crossing angle. Crossing points can be classified regarding the crossing angle of the airways involved, being the riskier crossing points the ones with lesser crossing angle. As explained in the scenario description, crossing angles are classified into three groups: crossing points with $\alpha<30^{\circ}$, crossing points with $30^{\circ}<\alpha<60^{\circ}$ and crossing points with $60^{\circ}<\alpha<90^{\circ}$. Columns 4,5 and 6 of Table 7 indicates the number of crossing points involved at each group. Overall, the number of crossing points with $\alpha<30^{\circ}$ predominates for all airways. This is an intrinsic characteristic of this airspace design that we cannot dealt with, but two airways set can be separated:

(a) From UQ57 to UQ500 we find the airways without initial traffic. Thus, the RPAS introduction means an increment of the crossing points and the conflict risk. However, we cannot find a relation between the increment of the crossing points and their angles because conflict-risk variations are very diverse depending on the airway the RPAS is introduced.

(b) The rest of the airways have initial traffic, so there is no increment on the number of crossing points neither modification of the crossing angles. This outcome implies that conflict-risk variations do not depend on the crossing angle once there is initial traffic.

Therefore, the results of the analysis of this conflict-risk factor are somewhat vague because no main conclusions can be extracted.
5.2.2.3. Airway length. This conflict-risk factor initially supposed a contradiction because the larger airway length, the minor should be the conflict risk. The last column of shows airway length classification: Short ( $<200 \mathrm{NM})$, Medium (200-400 NM) and Large ( $>400 \mathrm{NM})$. This conflict-risk factor results that airways with minor risk are shorter, which contradict our initial assumption. The reason is that this conflictrisk factor is not a proper indicator because it does not bear in mind the number of crossing points throughout the airway or the exposure time. Therefore, this factor should be modified in future works to provide appropriate safety information.

5.2.2.4. Airway density. This analysis aims to assess if the RPAS introduction has any influence or relation to the initial airway density. Airway density assessment is performed for an airway with high traffic (8,3\% airways), medium traffic (16,7\% airways) and low traffic (25\%), and the rest of airways maintains their initial traffic. The airways selected are: UN725 (4,1571 aircraft/hour), UP181 (1,4 aircraft/hour) and UM190 (0,0143 aircraft/hour). Moreover, we perform a sensitivity analysis with the RPAS average horizontal speed (250, 300 and 350 knots).

Overall, the introduction of RPAS implies a conflict-risk decrease by the horizontal speed increment, which confirms the results aforementioned, see Fig. 4. Mainly, RPAS introduction means that airway UM190 (low traffic) increases, UP181 (medium traffic) does not vary, and UN725 (high traffic) diminishes the conflict risk. These outcomes confirm a relation: if the number of RPAS is minor than the number of aircraft, the conflict risk diminishes; if the number of RPAS is similar to conventional aircraft, the conflict risk barely varies; and if the number of RPAS is minor than conventional aircraft, the conflict risk decreases. Nonetheless, we cannot ensure a linear relationship between the RPAS density introduced and initial traffic because it requires further work. Therefore, these outcomes conclude that the introduction of one RPAS through airways with initial traffic is affordable (conflict risk is minor than TLS), and does not depend on the initial airway density (although it may imply a capacity reduction). The reason is that crossing points are not increased, and the variation of speed barely affect.

\section{Conclusions}

This article assesses the impact of RPAS integration in non-segregated airspace based on a conflict-risk assessment methodology for airspace planning purposes. This novel research is comprised of the proposal of a framework, the development of a conflict-risk methodology and the identification of conflict-risk factors. The goal is twofold: to quantify the number of RPAS that can operate in an air traffic volume without exceeding a safety level, and to ascertain geometrical and operational factors that affect and may regulate the forthcoming

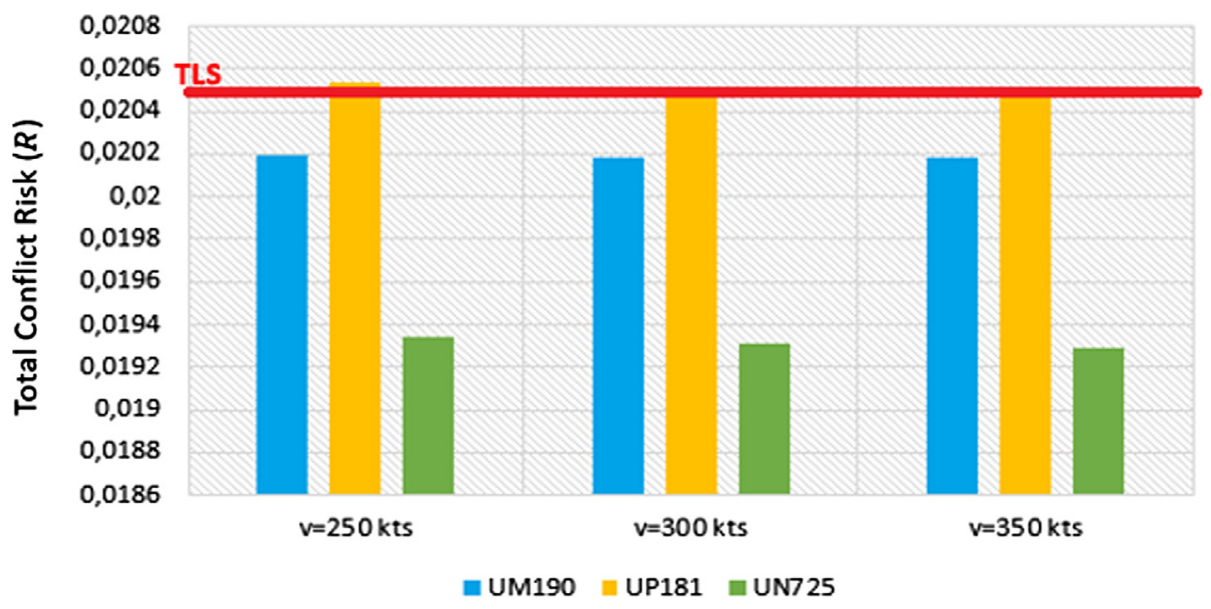

Fig. 4. Conflict risk depending on the airway density. 
RPAS integration. The methodology has been applied to en-route air traffic volume in Spain, characterising a limit of safety (TLS) based on the current operation and performing a sensitivity analysis of the RPAS average horizontal speed. Main conclusions of the research are:

- The framework and methodology developed allow detecting the maximum number of RPAS per airway that can be introduced in a sector without exceeding the TLS. Identifying the airways that permit a greater number of RPAS and which are more restrictive. However, this methodology does not permit to quantify the degree of affection for some operational factors, e.g., the number of crossing angles and the airway length.

- The most crucial conflict-risk factor is the number of crossing points in an air traffic volume, or specifically, the number of effective crossing points within. In this way, the introduction of RPAS through airways without initial traffic implies the increase of the conflict risk and may prevent RPAS integration. On the other hand, the introduction of RPAS through airways with initial traffic is allowed and, depending on the airway density, may reduce or increase the conflict risk.

- The results of the geometrical factors (crossing angle and airway length) are somewhat vague because no main conclusions can be extracted. It is evident that a crossing angle of $<30^{\circ}$ deteriorate and a crossing angle closer to $90^{\circ}$ favour the conflict risk. Likewise, airway length influences the conflict risk, but no conclusions can be extracted. Hence, these indicators should be removed from the methodology with others because they are extremely influenced by the airway density and exposure time.

- Operational factors (speed and airway density) provides diverse results. RPAS average horizontal speed is a crucial factor because we have concluded that conflict risk is lower when RPAS speed increases. However, airway density requires further research to assess whether there is a mathematical relation between the number of RPAS and the initial traffic removed or not.

This paper is one of the first attempts to quantify the impact of RPAS integration in non-segregated airspace. RPAS introduction is fairly challenging because it requires assessing different elements, factors and systems, and operational restrictions should be defined before its integration. Therefore, this work could be a meaningful standpoint to deepen the relations between safety-capacity and to ascertain operational restrictions. Nonetheless, this methodology presents the following limitations that should be revised in further works: it does not discriminate between strategical or tactical operations and does not allow mixing RPAS jointly with conventional aircraft in the same airway.

\section{Appendix A. Supplementary material}

Supplementary data associated with this article can be found, in the online version, at https://doi.org/10.1016/j.ssci.2018.08.018.

\section{References}

Alam, S., Shafi, K., Abbass, H.A., Barlow, M., 2009. An ensemble approach for conflict detection in Free Flight by data mining. Transp. Res. Part C Emerg. Technol. 17, 298-317. https://doi.org/10.1016/j.trc.2008.12.002.

Clothier, R., Clothier, R.A., Williams, B.P., Coyne, J., Wade, M., Washington, A., 2015. Challenges to the development of an airworthiness regulatory framework for unmanned aircraft systems challenges to the development of an airworthiness regulatory framework for unmanned aircraft systems describing the risk paradigm. In: 16th Australian Aerospace Congress. Melbourne, Australia.

Clothier, R., Walker, R., Fulton, N., Campbell, D., 2007. A casualty risk analysis for unmanned aerial system (UAS) operations over inhabited areas. Second Australas.
Unmanned Air Veh. Conf., pp. 1-15

Clothier, R.A., Palmer, J.L., Walker, R.A., Fulton, N.L., 2011. Definition of an airworthiness certification framework for civil unmanned aircraft systems. Saf. Sci. 49, 871-885. https://doi.org/10.1016/j.ssci.2011.02.004.

Clothier, R.A., Williams, B.P., Fulton, N.L., 2015b. Structuring the safety case for unmanned aircraft system operations in non-segregated airspace. Saf. Sci. 79, 213-228. https://doi.org/10.1016/j.ssci.2015.06.007.

Dalamagkidis, K., Valavanis, K.P., Piegl, L.A., 2008a. On unmanned aircraft systems issues, challenges and operational restrictions preventing integration into the National Airspace System. Prog. Aerosp. Sci. 44, 503-519. https://doi.org/10.1016/j.paerosci. 2008.08.001.

Dalamagkidis, K., Valavanis, K.P., Piegl, L.A., 2008. Evaluating the risk of unmanned aircraft ground impacts. In: 2008 Mediterr. Conf. Control Autom. - Conf. Proceedings, MED'08, pp. 709-716. https://doi.org/10.1109/MED.2008.4602249.

EASA, 2017. NPA 2017-05 (A): Introduction of a Regulatory Framework for the Operation of Drones. https://doi.org/Related A-NPA: 2015-10—RMT.0230-18.12.2015.

EASA, 2015. Advance NPA 2015-10: Introduction of a regulatory framework for the operation of drones.

EUROCONTROL, 2015. Approach to the introduction of RPAS in BADA 3 Aircraft Performance Model, EEC Technical Note No. 15/11/06-50. https://doi.org/10.1177/ $1090820 X 10391211$.

FAA, 2017. 8130.34D - Airworthiness Certification of Unmanned Aircraft Systems and Optionally Piloted Aircraft.

Geisinger, K.E., 1985. Airspace conflict equations. Transp. Sci. 19, 139-153. https://doi. org/10.1287/trsc.19.2.139.

ICAO, 2015. Manual on Remotely Piloted Aircraft Systems (Rpas). 2015.

ICAO, 2009. Unmanned Aircraft Systems (UAS) - Circular 328 AN/190, Cir 328 AN/190 https://doi.org/10.1016/B978-0-12-374518-7.00016-X.

ICAO, 2001. Doc 9574 - Manual on Implementation of a $300 \mathrm{~m}(1000 \mathrm{ft})$ Vertical Separation Minimum Between FL 290 and FL 410 Inclusive.

ICAO, 1998. Doc 9689-AN/953 - Manual on airspace planning methodology for the determination of separation minima. https://doi.org/10.1007/s13398-014-0173-7.2.

International Civil Aviation Organization, 1998. Doc 9689-AN/953 - Manual on airspace planning methodology for the determination of separation minima 1-205. https:// doi.org/10.1007/s13398-014-0173-7.2.

Lum, C., Waggoner, B., 2011. A Risk Based Paradigm and Model for Unmanned Aerial Systems in the National Airspace. Infotech@aerosp. 2011 1-31. https://doi.org/10. 2514/6.2011-1424.

Mehadhebi, K., Lazaud, P., 2004. A Synthesis of Current Collision Risk Models. ICAO Separation and Airspace Safety Panel, 5th Meeting of the Working Group of the Whole (SASP-WG/WHL/5-WP/9), Japan.

Melnyk, R., Schrage, D., Volovoi, V., Jimenez, H., 2014. A third-party casualty risk model for unmanned aircraft system operations. Reliab. Eng. Syst. Saf. 124, 105-116. https://doi.org/10.1016/j.ress.2013.11.016.

Netjasov, F., 2012a. Framework for airspace planning and design based on conflict risk assessment Part 1: conflict risk assessment model for airspace strategic planning. Transp. Res. Part C Emerg. Technol. 24, 190-212. https://doi.org/10.1016/j.trc. 2012.03.002.

Netjasov, F., 2012b. Framework for airspace planning and design based on conflict risk assessment. Part 2: Conflict risk assessment model for airspace tactical planning. Transp. Res. Part C Emerg. Technol. 24, 213-226. https://doi.org/10.1016/j.trc. 2012.03.003.

Netjasov, F., Janic, M., 2008. A review of research on risk and safety modelling in civil aviation. J. Air Transp. Manag. 14, 213-220. https://doi.org/10.1016/j.jairtraman. 2008.04.008.

Oswald, P., Hershey, W., 2007. Helping global hawk fly with the rest of us. In: ICNS Conference.

Pérez-Castán, J.A., Gómez Comendador, F., Arnaldo-Valdés, R.M., Hernández Gila, L. Torrecilla Puebla, J., 2017. Conflict-resolution algorithms for separation minima definition of rpas in a non-segregated airspace. In: 7th EASN International Conference on Innovation in European Aeronautics Research. Warsaw.

Persiani, C.A., Bagassi, S., 2013. Route planner for unmanned aerial system insertion in civil non-segregated airspace. Proc. Inst. Mech. Eng. Part G J. Aerosp. Eng. 227, 687-702. https://doi.org/10.1177/0954410012439975.

Reich, P.G., 1966a. Analysis of long-range air Separation Standards - II. J. Navig. 169-186. https://doi.org/10.1017/S0373463300047196.

Reich, P.G., 1966b. Analysis of long-Range air traffic systems - Separation Standards I. J. Inst. Navig. 50, 436-477.

Román-Cordón, R., Arnaldo-Valdés, R.-M., Cuerno-Rejado, C., Gómez Comendador, F. 2017. Integración de aeronaves pilotadas por control remoto en espacio aereo: diseño y desarrollo del interfaz de la estación de control en tierra. DYNA 93. https:// doi.org/https://doi.org/10.6036/8394.

Siddiqee, W., 1973. A mathematical model for predicting the number of potential conflict situations at intersecting air routes. Transp. Sci. 7, 571-577. https://doi.org/10. 1287/trsc.7.2.158.

Tang, J., Piera, M.A., Guasch, T., 2016. Coloured Petri net-based traffic collision avoidance system encounter model for the analysis of potential induced collisions. Transp. Res. Part C Emerg. Technol. 67, 357-377. https://doi.org/10.1016/j.trc.2016.03. 001. 